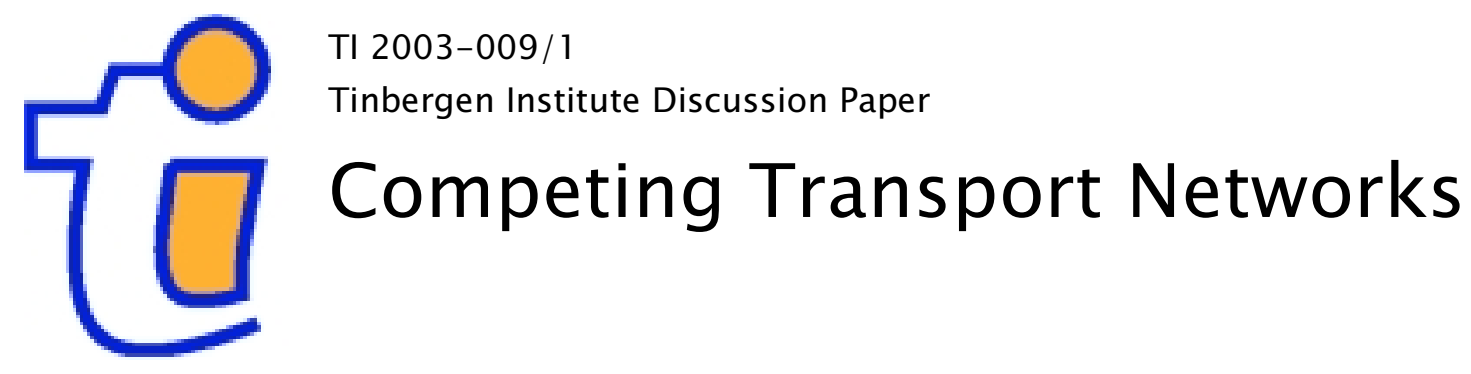

Marco J. van der Leij

Faculty of Economics, Erasmus University Rotterdam, and Tinbergen Institute 
Tinbergen Institute

The Tinbergen Institute is the institute for economic research of the Erasmus Universiteit Rotterdam, Universiteit van Amsterdam and

Vrije Universiteit Amsterdam.

Tinbergen I nstitute Amsterdam

Keizersgracht 482

1017 EG Amsterdam

The Netherlands

Tel.: +31.(0)20.5513500

Fax: $\quad+31 .(0) 20.5513555$

Tinbergen Institute Rotterdam

Burg. Oudlaan 50

3062 PA Rotterdam

The Netherlands

Tel.: $\quad+31 .(0) 10.4088900$

Fax: $\quad+31 .(0) 10.4089031$

Most TI discussion papers can be downloaded at

http://www.tinbergen.nl 


\title{
Competing Transport Networks
}

\author{
Marco J. van der Leij* \\ Tinbergen Institute \\ Erasmus University Rotterdam
}

16 January, 2003

\begin{abstract}
In a circular city model, I consider network design and pricing decisions for a single fast transport connection that faces competition from a slower but better accessible transport mode. To access the fast transport network individuals have to make complementary trips by slow mode. This fact has interesting implications on the location decisions. I show that in the presence of competition the profitmaximizing and socially optimal decision would be to cluster the two stations. By contrast, in the absence of competition both a profit-maximizing firm and a social planner would locate the two stations on opposite sides of the circle.
\end{abstract}

Key words: Networks, Transportation, Competition, Market area, Circular city.

*I am grateful to Sanjeev Goyal, Jose Luis Moraga-Gonzalez and Andrea Galeotti for their comments and suggestions. I also thank the seminar audience at the Tinbergen Institute for their comments. Address for correspondence: Marco van der Leij, Tinbergen Institute H16-34, Erasmus University Rotterdam, Burg. Oudlaan 50, 3062 PA Rotterdam, The Netherlands, e-mail: mvanderleij@few.eur.nl 


\section{Introduction}

In many countries privatization and deregulation of transport industries raises a lot of opposition. One of the greatest fears is that a privatized transport firm would change its network structure, that is, the location of their stations and the connections between cities, such that some cities would be deprived of a good connection to other cities. Indeed the events after the deregulation of the U.S. airline industry in 1978 have shown that this is not unlikely. Since 1978 almost all airline carriers have transformed their U.S. networks into hub-and-spoke networks. As a result, most airline passengers can not reach their destination directly. Moreover, the hub-and-spoke network structure has raised concerns on market dominance at hub airports ${ }^{1}$.

Although the emergence of hub-and-spoke networks has been analyzed extensively, the airline literature typically ignores the presence of competing transport modes. This approach is reasonable in the case of the U.S. airline industry, as the distance between cities in the U.S. is large and the railway industry in the U.S. does not offer a feasible alternative to airline travel. However, in Europe and Japan the situation is different due to the availability of a network of high-speed train connections. Furthermore, competition from other transport modes is even more important in other transport industries. For example, railway transport has always faced fierce competition from transport by car, since the speed of a train and a car is comparable. In urban areas, underground transport faces competition from bus and tram services. One should note that these competing transport networks differ in speed and accessibility. Typically the faster transport mode is less accessible than the slower transport mode.

With the current wave of deregulation in other transport industries than the airline industry, it is necessary to understand network design decisions in case a transport net-

\footnotetext{
${ }^{1} \mathrm{~A}$ hub-and-spoke network is a network in which one airport, the hub, has direct connections to all other airports, while all other airports, the spoke airports, are only connected to the hub airport. Hendricks, Piccione \& Tan (1995) show that under economies of density either the empty network, the hub-and-spoke network or the point-to-point network is optimal. Hendricks, Piccione \& Tan (1997) show that a hub-and-spoke network may prevent entry into a hub-to-spoke market. Borenstein (1989) shows that route and airport dominance enable carriers to raise prices.
} 
work operator faces competition from other transport services. In this paper I therefore introduce a model in which I consider the station locations and the price a network operator would charge, when individuals also have the alternative to travel through a slower but better accessible competing transport network.

The model is simple but reasonable, and it provides interesting results and intuition for the role of competing transport networks in network design. Individuals live and work at random locations on a circular city and they need to travel between their home and work. Each combination of home location and work place, therefore, constitutes a trip, having an origin and a destination. An individual can make a trip by a slow transport mode around the circle, either clockwise or counterclockwise. Alternatively he or she can use the fast transport system to cross the circle. It is crucial that slow mode transport is necessary to access the fast transport network. Hence, a trip by fast mode consists of three complementary journeys; a trip by slow mode from home to the departure station, a trip by fast mode from station to station, and finally a trip by slow mode from the terminating station to the work place. In this model I first derive demand for the fast transport connection. For that purpose I derive the size of the market area for the fast connection, which I define as the set of trips for which a fast transport connection is preferred. This definition differs from the usual interpretation of a market area as being a region around a station.

Next, I derive the main result of the paper. I consider location and pricing decision in case the fast transport connection is operated by a profit-maximizing firm. Contrary to intuition, I show that the stations of the fast connection are not located on opposite sides of the circle but somewhat closer. Hence, the monopolist typically clusters the stations. This tendency of clustering becomes stronger if competition from the slow transport mode is stronger. In an extreme case, when there is little difference between the velocity of a fast connection and a slow connection, the distance between the two stations is only one sixth of the circle (Figure 1). On the other hand, in the absence of competition the stations are located symmetrically on the circle. I also consider the socially optimal locations of the two stations. Again, the stations are not located on opposite sides of the circle. However 


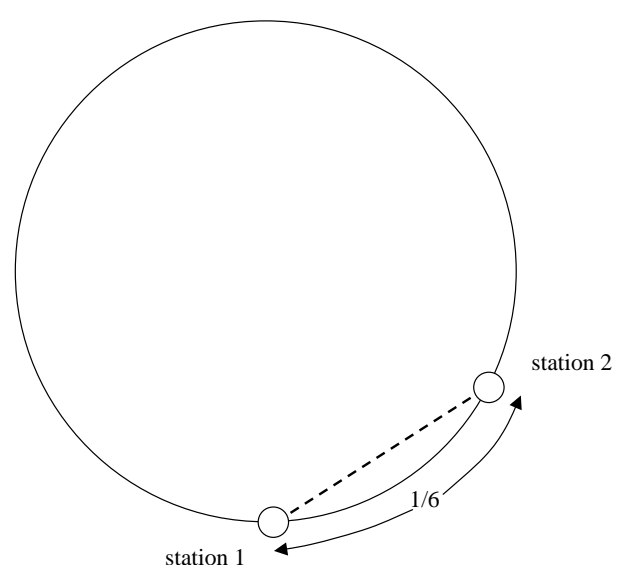

Figure 1: A fast transport network in the circular city where the distance between the stations is $1 / 6$ of the circle.

the distance between the socially optimal locations is larger than the distance between the monopolist's optimal locations, since a social planner is also concerned about the benefit from a trip. The intuition behind these results is that competition is more severe for trips whose origin and destination is between the two stations, than for trips whose origin and destination is in the hinterland of the two stations. In case the stations are located on opposite sides of the circle, there is no hinterland and competition creeps in from both sides of the circle. Hence, the firm has an incentive to cluster the stations such that it obtains an area where competition is absent.

Literature that considers location decisions of transport networks is sparse. The model in this paper is indeed the first model that introduces competition between transport networks of different speed and density to analyze the impact on location decisions. There is some related literature. Braid (1989) uses the linear city model to find the optimal locations of bridges across a river, where individuals live on one side of the river and work on the other side. Also, Crampton (2000) uses the linear city model to compute optimal urban-rail station spacing. However, in these two papers it is assumed that transportation across a bridge or by train occurs instantaneously without any costs, while in my paper individuals still face transportation costs and a fare that are increasing in distance when they use a fast transportation connection. This difference is crucial in 
the analysis of the design of the transport network. There is also some literature that consider the market areas that competing transport firms serve, when the transportation costs between transport modes differ (Hyson \& Hyson 1950, Niérat 1997). However, in this literature the network structure is given, while in this paper decisions on the network design are the primary focus of the analysis.

The structure of the paper is as follows. In Section 2 I lay out the model and I derive the demand function for the fast transport connection. In Section 3 I assume that the fast transport network is operated by a profit-maximizing firm, and I derive the optimal fare and station locations for the firm. Next, in Section 4, I assume that a social planner chooses the location of the two stations and the optimal fare. Finally, in Section 5 I conclude.

\section{The model}

In this section I describe the transportation model. Infinitesimal individuals are uniformly distributed with density 1 on a circle $\mathcal{C}$ of unit circumference. All individuals travel to one random destination that is also uniformly distributed on the circle. This destination is independent of the location of the individuals. Together, the set of individual's locations and the set of destinations form the set of trips $\mathcal{T}=\mathcal{C} \times \mathcal{C}$.

To make a trip, an individual can choose between two transport modes, a 'slow' transport mode or a 'fast' transport mode. I assume that the slow mode is offered competitively at price 0 , and that all individuals can access the slow mode network directly from their location. Furthermore, I assume that the time costs are linear in distance, and that every trip yields a value $u$ to the individual. Hence an individual on a trip $(x, y) \in \mathcal{T}$ obtains a utility of

$$
U_{S}(x, y)=u-g|y-x|
$$

if he chooses the slow mode. Here $g>0$ is the marginal time cost of travelling by slow mode, and the distance $|y-x|$ is the shortest arc length between $x$ and $y$. Note that the longest distance a traveller could make is a trip where the destination is opposite to the 
starting point. Hence, the utility of a trip is bounded from below by $U_{S}\left(x, \frac{1}{2}\right)=u-g / 2$. I assume that $u$ is high enough to persuade all individuals to travel, that is, $u \geq g / 2$.

On the other hand, an individual might choose the fast mode for her trip. I assume that the fast mode network consists of only one (two-way) connection between two stations, located at $z_{1}$ and $z_{2}$ on the circle. Hence, only individuals on a trip $\left(z_{1}, z_{2}\right)$ (or $\left(z_{2}, z_{1}\right)$ in the opposite direction) can access their destination directly through the fast mode network. All other individuals have to travel by slow mode first to and then from the stations to complete a trip. I therefore assume that the utility of a trip $(x, y) \in \mathcal{T}$ to an individual $x$ travelling to $y$ by fast mode is

$$
U_{F}(x, y ; d, p)=u-f d-p-g\left|z_{i}-x\right|-g\left|y-z_{j}\right|
$$

where $i, j \in\{1,2\}, i \neq j$ are chosen such that $\left|z_{i}-x\right|+\left|y-z_{j}\right|$ is minimal. Here $d \equiv\left|z_{2}-z_{1}\right|$ is the distance between the two stations, $f$ is the marginal time cost of travelling on the fast mode connection, $0<f<g$, and $p \geq 0$ is the fare (price) of the fast connection.

In Figure 2 I illustrate the transportation costs of the two travel mode options for a trip from $x$ to $y$, where the upper and lower half of the figure only differ in the destination $y$. The solid arrow in the two pictures illustrates a trip from $x$ to $y$ by slow mode. The transportation cost of such a trip is $g|y-x|$. On the other hand, the three dotted arrows illustrate a trip from $x$ to $y$ by fast mode, which consists of three different journeys with a total cost of $g\left|z_{1}-x\right|+f d+p+g\left|y-z_{2}\right|$. Note that for the trip $(x, y)$ in the bottom picture an individual incurs a cost for a journey from $z_{2}$ to $y$ both for the fast mode option as for the slow mode option.

\subsection{Demand}

I now derive the demand for fast mode transport when prices and station locations are given. The procedure is similar to that in spatial models. However, derivation of the market area is more complicated, because the utility an individual obtains depends both on the location of the individual as on its destination. Hence, for each home location 


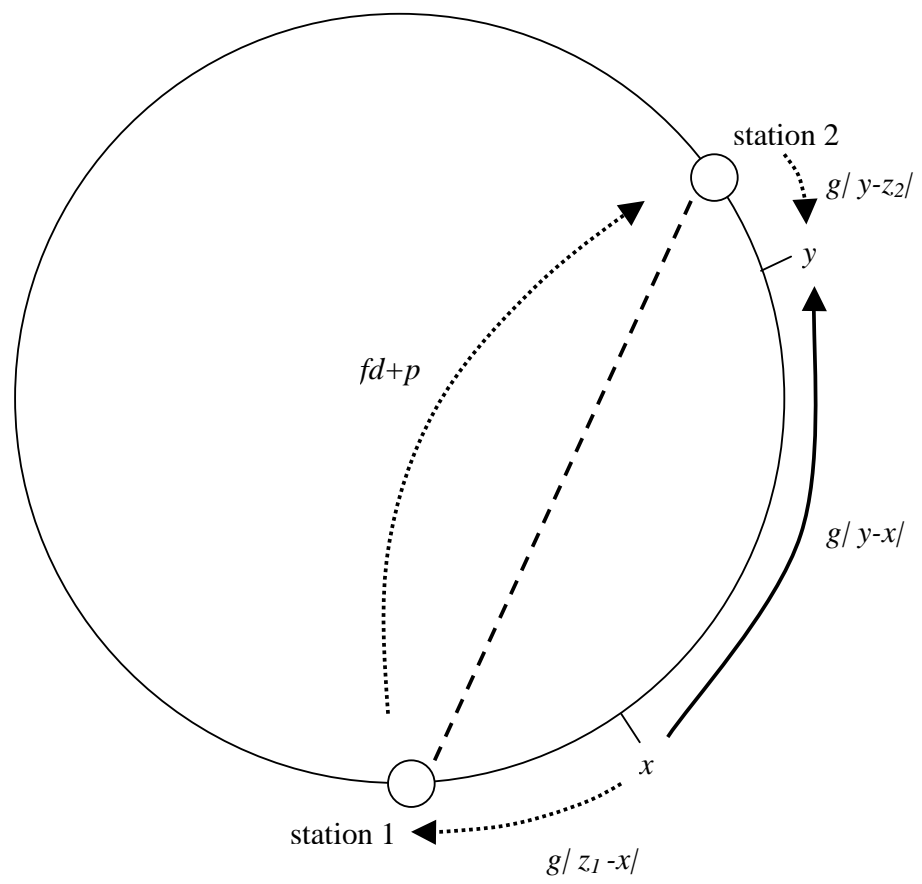

a) the destination is between the stations

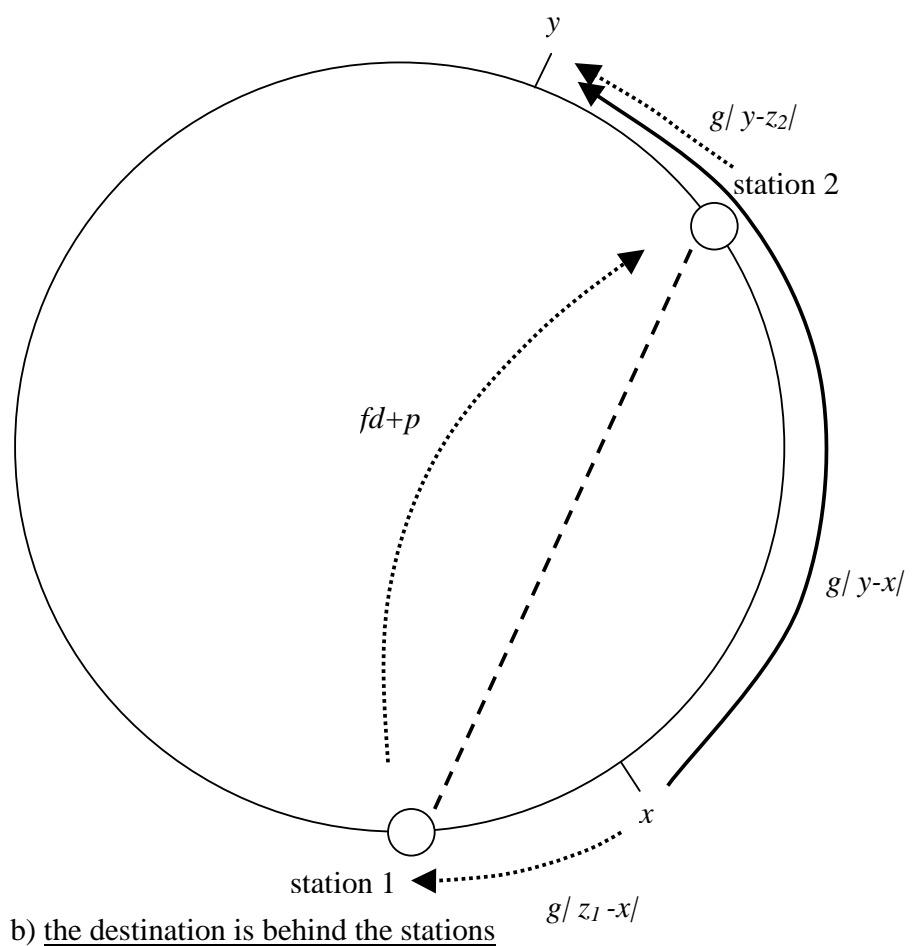

Figure 2: Transportation costs in two competing transport networks. 
there is a different region of workplaces for which the fast mode is preferred. Moreover, it turns out that the market area is not lying symmetrically around a station. I therefore introduce the following notation. I define a location $x$ to be between the stations if $x$ lies on the shortest arc length between $z_{1}$ and $z_{2}$, such that $\left|z_{2}-x\right|+\left|x-z_{1}\right|=d$. On the other hand, a location $x$ is behind the stations if $x$ lies on the longest arc length between $z_{1}$ and $z_{2}$, such that $\left|z_{2}-x\right|+\left|x-z_{1}\right|=1-d$. Note that if $z_{1}$ and $z_{2}$ are on opposite sides of the circle $x$ is both between and behind the stations.

First I assume that an individual on a trip $(x, y) \in \mathcal{T}$ prefers the fast mode if and only if

$$
U_{F}(x, y ; d, p) \geq U_{S}(x, y)
$$

or equivalently

$$
|y-x|-\left|z_{i}-x\right|-\left|y-z_{j}\right| \geq \frac{f d+p}{g} .
$$

for $i, j$ such that $\left|z_{i}-x\right|+\left|y-z_{j}\right|$ is minimal. It follows that an individual will only choose the fast transport mode if the fast transport connection offers a 'shortcut' to transportation by slow mode, that is, travelling from home to station $i$ and then from station $j$ to the workplace involves a shorter distance than travelling directly from home to work. Note that it immediately follows that the distance of the trip $|y-x|$ can not be too small. Hence, there are always trips where travel by slow mode is preferred.

Let me now consider the market area for the fast mode, which is the set of trips $\mathcal{T}_{F} \subset \mathcal{T}$ where the fast mode connection is preferred to the slow mode network. This area can be split into four parts. First I consider the trips $(x, y) \in \mathcal{T}_{F}$, where both $x$ and $y$ are located between the stations (Figure $2 a$ ). Because the stations are chosen such that $\left|z_{i}-x\right|+\left|y-z_{j}\right|$ is minimal, it must hold that $\left|z_{i}-x\right|+|y-x|+\left|y-z_{j}\right|=d$. Substituting into (1) and rewriting, it becomes clear that the fast mode is preferred as long as

$$
\left|z_{i}-x\right|+\left|y-z_{j}\right| \leq \frac{1}{2 g}(g d-f d-p) .
$$

It immediately follows that both $\left|z_{i}-x\right| \leq(g d-f d-p) / 2 g$ and $\left|y-z_{j}\right| \leq(g d-f d-p) / 2 g$.

In the second case, suppose that $x$ is between the stations but the destination $y$ is behind the stations (Figure $2 \mathrm{~b}$ ). Since the distance $|y-x|$ is the length of the shortest 
path between $x$ and $y$, it holds that

$$
|y-x|=\min \left\{\left|y-z_{i}\right|+\left|z_{i}-x\right|, \quad\left|y-z_{j}\right|+\left|z_{j}-x\right|\right\}
$$

Substituting into (1) the decision criterium becomes

$$
\begin{aligned}
& \left|y-z_{i}\right|-\left|y-z_{j}\right| \geq \frac{f d+p}{g} \text { and } \\
& \left|x-z_{j}\right|-\left|x-z_{i}\right| \geq \frac{f d+p}{g} .
\end{aligned}
$$

Because $x$ is between the stations, and $y$ is behind, (3) and (4) can be rewritten as

$$
\left|y-z_{j}\right| \leq \frac{1}{2}-d+\frac{1}{2 g}(g d-f d-p)
$$

and

$$
\left|x-z_{i}\right| \leq \frac{1}{2 g}(g d-f d-p)
$$

Given some starting point between the stations, $x$, cases 1 and 2 define a range of destinations for which the fast mode is preferred. This range is drawn in Figure 3 a. The size of the range is

$$
1 / 2-d+2 \hat{x}-\left|z_{i}-x\right|
$$

where

$$
\hat{x} \equiv \hat{x}(p, d) \equiv \frac{1}{2 g}(g d-f d-p)
$$

Consider now the third case, where $x$ is behind but $y$ is between the stations. This case is equivalent to the second case, except that starting and destination points are interchanged. Hence, in this case, $(x, y) \in \mathcal{T}_{F}$ if and only if

$$
\left|x-z_{i}\right| \leq \frac{1}{2}-d+\hat{x}
$$

and

$$
\left|y-z_{j}\right| \leq \hat{x}
$$

The fourth and last case considers a trip $(x, y)$ where both $x$ and $y$ are behind the stations. In this case

$$
|y-x|=\min \left\{1-d-\left|y-z_{j}\right|-\left|z_{i}-x\right|, \quad d+\left|y-z_{j}\right|+\left|z_{i}-x\right|\right\} .
$$




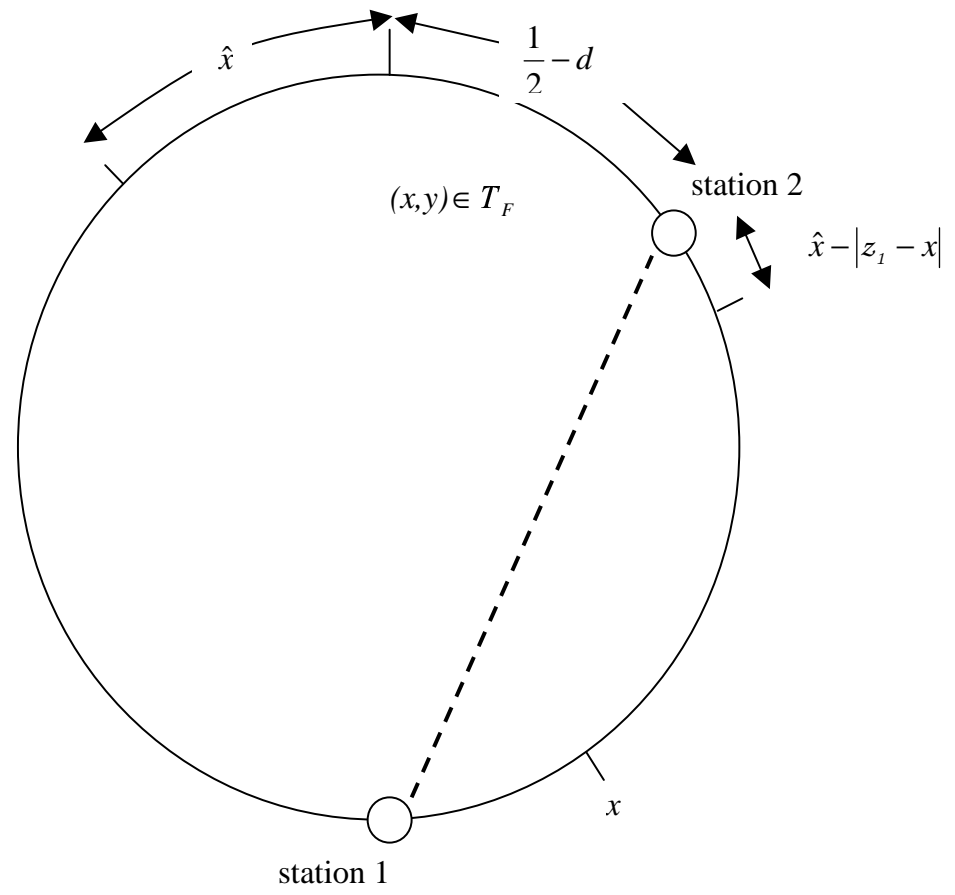

a) $\underline{x \text { is between the stations }}$

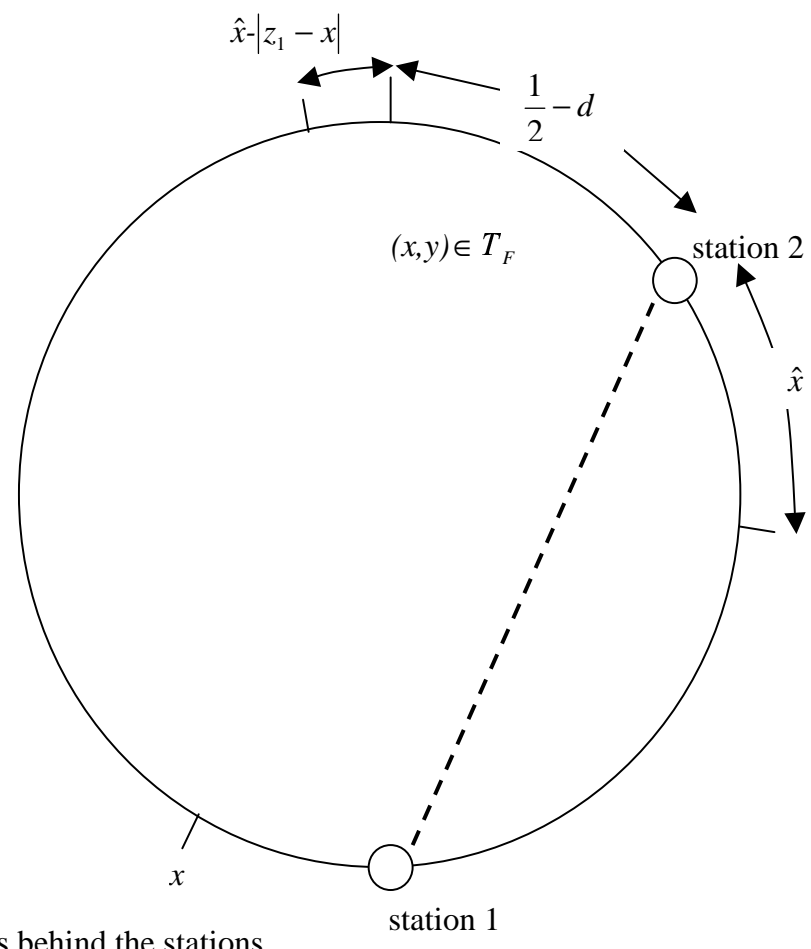

b) $x$ is behind the stations

Figure 3: Range of destination for which the fast mode is preferred, given some starting point, $x$. 
Substituting (9) into (1) and rewriting, the condition for $(x, y) \in \mathcal{T}_{F}$ becomes

$$
\left|z_{i}-x\right|+\left|y-z_{j}\right| \leq \frac{1}{2}-d+\hat{x}
$$

and

$$
\hat{x} \geq 0
$$

Cases 3 and 4 also constitute a range of destinations for which the fast mode is preferred whenever the starting point $x$ is behind the stations. This range is shown in Figure $3 \mathrm{~b}$. Again, the size of this range is $1 / 2-d+2 \hat{x}-\left|z_{i}-x\right|$.

Now that I have found the market area, I can derive the demand function. The following proposition states the demand function.

Proposition 1 Consider the transportation model of Section 2. If the distance between the stations is $d$, and the fare of a fast mode connection is $p$ with $p \leq g d-f d$, then demand is given by

$$
D(p, d)=6 \hat{x}^{2}+6 \hat{x}(1 / 2-d)+(1 / 2-d)^{2},
$$

where

$$
\hat{x} \equiv \hat{x}(p, d) \equiv \frac{1}{2 g}(g d-f d-p) .
$$

If $p>g d-f d$, then $D(p, d)=0$.

Proof: Consider a trip $(x, y) \in \mathcal{T}_{F}$, and suppose that $p \leq g d-f d$, such that $\hat{x} \geq 0$. From (2) and (5) it follows that if $x$ is between the stations then $\left|z_{i}-x\right| \leq \hat{x}$. On the other hand, if $x$ is behind the stations, equations (7) and (10) imply that $\left|z_{i}-x\right| \leq 1 / 2-d+\hat{x}$. Moreover, for a trip originating at $x$ the size of the range of destinations $y$, such that $(x, y) \in \mathcal{T}_{F}$, is

$$
1 / 2-d+2 \hat{x}(p ; d)-\left|z_{i}-x\right|
$$

where $\hat{x} \equiv \hat{x}(p ; d)$ is given in (6). Hence, because there are two stations, demand is given 


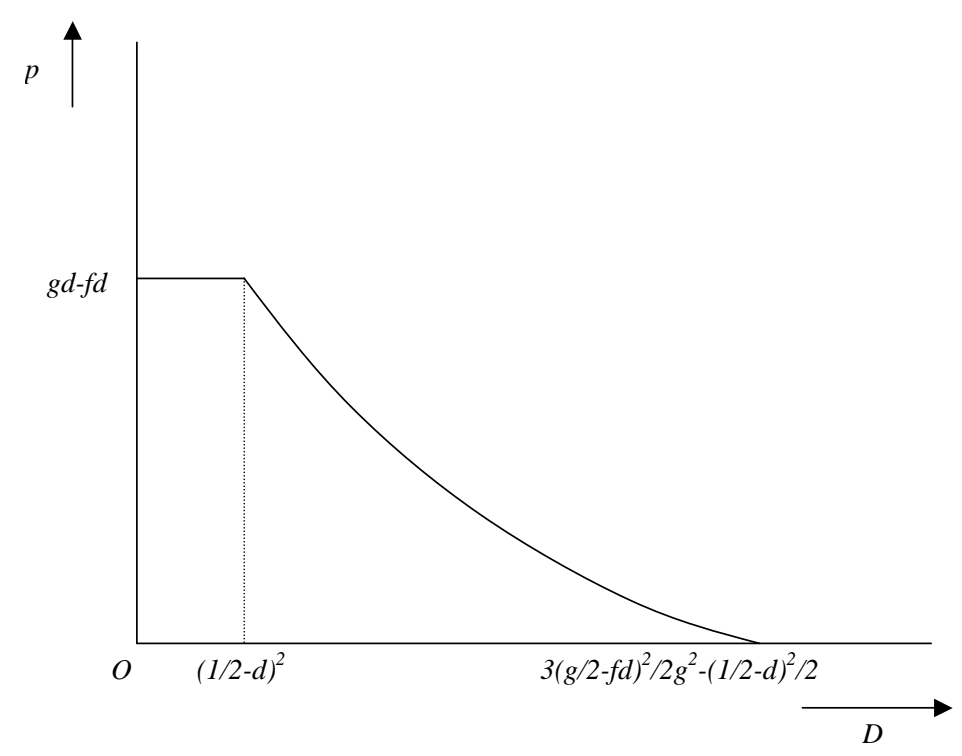

Figure 4: Demand function in the transport model.

by

$$
\begin{aligned}
D(p, d)= & 2 \int_{0}^{\hat{x}} 1 / 2-d+2 \hat{x}-x d x \\
& +2 \int_{0}^{\hat{x}+1 / 2-d} 1 / 2-d+2 \hat{x}-x d x \\
= & 6 \hat{x}^{2}+6 \hat{x}(1 / 2-d)+(1 / 2-d)^{2} .
\end{aligned}
$$

Furthermore, if $p>g d-f d$, then $\hat{x}<0$, and one of the conditions (2), (5), (8) or (11) is violated. Hence, if $p>g d-f d$, then the set $\mathcal{T}_{F}$ is empty. Q.E.D.

Figure 4 shows a typical demand function. The demand function is a quadratic function in $p$ and $d$, and for all feasible prices $0 \leq p \leq g d-f d$ the demand function is downward sloping and convex in $p$. Hence, the demand function has a familiar shape, except that there is an upperbound for $p$. If $p=g d-f d$, then

$$
D(g d-f d, d)=(1 / 2-d)^{2},
$$

while demand collapses to zero if $p$ becomes larger than $g d-f d$. 


\section{Profit maximizing location and pricing}

In this section I consider the optimal price and location in case the fast mode network is operated by a monopolist. To focus on the demand side I assume that a firm operating the connection incurs no costs.

I derive the optimal price and location for the monopolist operator. The monopolist maximizes profits

$$
\pi(p, d)= \begin{cases}p D(p, d) & \text { if } 0 \leq p \leq g d-f d \\ 0 & \text { otherwise }\end{cases}
$$

Since the fast mode's comparative advantage is greater the larger is $d$, one would expect that the optimal location of the two stations for the monopolist would be such that the stations are located on the opposite site of the circle, such that $d=1 / 2$. However, the following proposition shows that this is not true.

Proposition 2 Consider the distance $d^{M}$ between the two stations and price $p^{M}$ that maximize monopoly profits. The optimal price is

$$
p^{M}=\frac{g-f}{6}
$$

and the optimal distance is $d^{M}=1 / 6$ for $f<g \leq 3 f$, and

$$
d^{M}=\frac{g^{2}-f^{2}-2 g f}{2 g^{2}-6 f^{2}}
$$

for $g \geq 3 f$.

The proof follows from standard constrained optimization and is given in the Appendix. Note that $d^{M}$ is continuous in $g$, even at $g=3 f$, and that $d^{M} \rightarrow 1 / 2$ whenever $g \rightarrow \infty$.

Why do the stations not lie on opposite sides of the circle? The answer is hidden in the nature of competition in this transport model. Competition between the two transport modes is more severe for trips starting and ending between the two stations. In this case the length of the trip $|y-x|$ is typically smaller than the distance between the two stations (Figure 2a). For these trips, the slow mode becomes an attractive alternative if the price of the fast mode increases or if the start or destination of the trip is located further away from the stations. 
However, the fast mode network virtually does not face any competition for trips between the station's hinterlands, which are the locations behind the two stations. The reason is simple. Suppose that for a trip to station $2,\left(x, z_{2}\right)$ the fast mode is optimal. If the destination of a trip $(x, y)$ is further away from $x$ than station 2 (Figure $2 \mathrm{~b}$ ), an additional travel distance has to be covered. However, this additional travel distance is in both cases made by slow mode transport and therefore the additional travel costs does not depend on the choice of transport mode. Hence, if fast mode transport has a comparative advantage for a trip to station 2, it also has a comparative advantage for all trips to the hinterland of station 2. Clearly the size of the hinterland weighs heavily on the monopolist's demand. This can also be seen from Figure 3. Demand from the hinterland is however bounded, as it becomes attractive to use the slow mode network in the opposite direction, if the destination is located far away in the hinterland.

It now becomes clear why demand is not maximal when the stations are located on opposite sides of the circle. In this case the stations lack a big hinterland, as the fast mode network faces competition from the slow mode network from both sides of the circle. Only if the stations are located closer, the fast mode network is able to create and serve a hinterland, where competition from the slow mode network is less severe. Following this reasoning, one might think that it is optimal to minimize the distance of the stations. However, if the distance becomes smaller, the reservation price for a trip from station to station, $g d-f d$, decreases. At some point, a smaller distance between the two stations has to be combined with lower prices, which has a negative impact on profits, and as a result the optimal distance is bounded from below.

Note that the above result depends on the competitiveness of the transport modes. As $g$ increases or if $f$ decreases the slow mode becomes less competitive, and therefore there is less reason for the fast network operator to take competition from slow mode transport into account. Therefore, if $g \rightarrow \infty$ or if $f \rightarrow 0$, then the distance between the stations goes to a half. 


\section{Social welfare}

Since the optimal station locations from a monopolist's view are quite surprising, one wonders if results differ when the firm is operated by a social planner. Therefore, I turn to the question what price a social planner would set, and what station locations he would choose. I assume that a social planner maximizes the social welfare function

$$
W(p ; d)=\pi(p ; d)+C S(p ; d)
$$

where $C S(p ; d)$ is aggregate consumer surplus.

Consumer surplus has a simple structure. Consider first $C S^{S}$, the consumer surplus in case all travellers choose the slow mode, that is if $p>g d-f d$. Then

$$
C S^{S}=2 \int_{0}^{1 / 2} u-g x d x=u-\frac{1}{4} g .
$$

If $p<g d-f d$, such that some travellers choose the fast mode, the consumer surplus must be higher than $C S^{S}$. This extra surplus that travellers obtain from using the fast mode network is given by the usual consumer surplus triangle under the demand function $D(p ; d)$. That is

$$
C S(p, d)=C S^{S}+\int_{p}^{g d-f d} D(s ; d) d s
$$

and one can apply the usual social welfare analysis in a partial equilibrium model. Hence, at the social optimum, price equals marginal cost, that is $p^{S}=0$, and social welfare is given by

$$
\begin{aligned}
W(0 ; d) & =0+C S^{S}+\int_{0}^{g d-f d} 6\{\hat{x}(p, d)\}^{2}+6 \hat{x}(p, d)(1 / 2-d)+(1 / 2-d)^{2} d p \\
& =C S^{S}+2 g \int_{0}^{(g d-f d) / 2 g} 6 x^{2}+6 x(1 / 2-d)+(1 / 2-d)^{2} d x \\
& =u-g / 4+2 g x_{0}\left(2 x_{0}^{2}+3 x_{0}(1 / 2-d)+(1 / 2-d)^{2}\right),
\end{aligned}
$$

where $x_{0}=(g d-f d) / 2 g$.

I now turn to the socially optimal distance between the stations, $d^{S}$. The first order derivative of the welfare function is given by

$$
\frac{\partial W(0 ; d)}{\partial d}=-6 f x_{0}^{2}+(2 g-6 f) x_{0}(1 / 2-d)+(g-f)(1 / 2-d)^{2} .
$$


Note that if $d=0$, then $\partial W / \partial d=(g-f) / 4>0$, and if $d=1 / 2$, then $\partial W / \partial d=-6 f x_{0}^{2}<$ 0 . Since the welfare function is a cubic function in $d$, the optimal distance $d^{S}$ must be between 0 and $1 / 2$. Solving $\partial W / \partial d=0$ after some manipulations, it turns out that the only optimal solution is

$$
d^{S}=\frac{g}{g+3 f+\sqrt{g^{2}+3 f^{2}}} .
$$

It is easy to check that $d^{S}>1 / 6$ for $g>f$. Furthermore

$$
d^{S}>\frac{g}{2 g+3 f}>\frac{g^{2}-f^{2}-2 g f}{2 g^{2}-6 f^{2}}=d^{M} .
$$

Hence, I have the following proposition

Proposition 3 For all $g>f$, the distance between the two stations that maximizes social welfare, $d_{S}$, and the distance between the stations that maximizes firm's profits, $d_{M}$, are such that

$$
d^{M}<d^{S}<1 / 2
$$

So a social planner would not locate the stations on opposite sides of the circle either. This is in line with the monopolist's decision. However, from the point of view of the social planner, the monopolist separates the stations too little. The reason is as follows. A social planner is not only concerned about the level of demand for the fast connection, but also about the utility individuals obtain. This utility depends on the reduction in transport costs due to availability of a fast transport connection. The individual that benefits the most from the fast mode connection is an individual making a trip from station to station, $\left(z_{i}, z_{j}\right)$, in which case the cost reduction is

$$
U_{F}\left(z_{1}, z_{2} ; d, 0\right)-U_{S}\left(z_{1}, z_{2}\right)=g d-f d .
$$

Hence, the greater the distance between the stations, the higher is this maximum cost reduction, and this effect pushes the socially optimal distance, $d^{S}$, away from the monopolist's optimal distance, $d^{M}$.

That the socially optimal distance between the stations is still smaller than a half is not directly obvious given that the maximum cost reduction is increasing in $d$. The reason 
of this result lies in the fact that more individuals obtain the maximum cost reduction if $d$ gets smaller. In fact all individuals making a trip from the hinterland of station $i$ to the hinterland of station $j$ obtain the maximal cost reduction of $g d-f d$, as long as the stations are between the origin and destination of the trip. That is, if $(x, y)$ is such that $|y-x|=\left|z_{i}-x\right|+d+\left|y-z_{j}\right|$ then

$$
U_{F}(x, y ; d, 0)-U_{S}(x, y)=g d-f d .
$$

The reason is already explained in the monopoly case. If the fast mode has a comparative advantage on a trip from station to station, it also has the same comparative advantage on a trip from the hinterland of a station to the hinterland of the other station since travelling behind a station is always made by slow mode. Hence the cost of travelling behind a station adds up to the transportation costs whatever transport mode is chosen. If $d=1 / 2$ then the stations lack a big hinterland. In fact, if $d=1 / 2$, then the maximum cost reduction in only obtained for a trip from station to station, and consequently the cost reduction effect becomes second order. Hence, social welfare is not optimal if $d=1 / 2$.

\section{Conclusion}

In this paper I have shown that a simple transportation model with competing transport modes can give interesting insights into the optimal network design of a transport network. Competition from a slower, but more accessible transport network incites a monopolist transport operator not to locate the stations on opposite sides of the circle, as the size of the hinterland increases if the stations are located closer to each other. This incentive to diminish the distance between stations is also present for a social planner; however, it is stronger for a monopolist than for a social planner. On the other hand, if the difference in speed between the slow and the fast mode is very large, competition is not an issue and the fast mode operator locates its stations near opposite sides of the circle.

Of course, the model I have presented uses strong assumptions and future research should try to generalize and extend this restricted model to analyze a broader range of issues in transport economics. To my opinion, the most interesting extension would be to 
solve the transportation model for $n$ stations and endogenize the number of stations and connections. Such an extension could be used to analyze the impact of new technologies on the density and the structure of transportation networks. To see the relevance of such issues, consider the history of railway industries. In the beginning of the 20th century railway transport was by far the most efficient transport method for longer distances. However, in course of the 20th century it has lost its comparative advantage to airplane and car. This has resulted in a reduction of stations and connections in the railway network. Nowadays, the railway industry is coming back with the introduction of highspeed trains, reviving the competition between airline and railroad travel. It is not clear what the implications on airline networks are. A model of competing transport networks with an endogenous number of stations could shed more light on this issue.

\section{A Appendix}

Proof of Proposition 2: For the moment, allow $d=0$ in the feasible set $\mathcal{F}$, such that

$$
\mathcal{F}=\left\{(p, d) \in \mathbb{R}^{2} \mid 0 \leq d \leq 1 / 2 ; 0 \leq p \leq g d-f d\right\}
$$

Then the monopolist solves the optimization problem

$$
\begin{array}{ll}
\max _{p \geq 0, d \geq 0} & p D(p, d) \\
\text { s.t. } & p \leq(g-f) d \\
\text { and } & d \leq 1 / 2
\end{array}
$$

Note that the feasible set $\mathcal{F}$ is closed and bounded with linear restrictions, and that the profit function $p D(p, d)$ is continuously differentiable at $p$ and $d$. Hence, for any value of $f$ and $g, 0<f<g$, a maximum exists and the optimal solution satisfies the Kuhn-Tucker first order conditions.

It is easy to check that $d>0$ and $p>0$. Hence the first order conditions are given by

$$
\begin{gathered}
p \frac{\partial D(p ; d)}{\partial p}+D(p ; d)-\lambda=0 \\
p \frac{\partial D(p ; d)}{\partial d}+\lambda(g-f)-\mu=0,
\end{gathered}
$$




$$
\begin{gathered}
p \leq(g-f) d \text { and } \lambda(g d-f d-p)=0, \\
d \leq 1 / 2 \text { and } \mu(1 / 2-d)=0,
\end{gathered}
$$

for some $\lambda \geq 0$ and $\mu \geq 0$. Remember that $\hat{x} \equiv \hat{x}(p ; d) \equiv \frac{1}{2 g}(g d-f d-p)$. Conditions (13) and (14) can be rewritten as

$$
\lambda=18 \hat{x}^{2}+\frac{1}{g}(18 g-6 f) \hat{x}(1 / 2-d)+\frac{1}{g}(4 g-3 f)(1 / 2-d)^{2}-3 \frac{g-f}{2 g}(2 \hat{x}+1 / 2-d),
$$

and

$$
\mu=\lambda(g-f)+\frac{p}{g}\{(g-3 f)(1 / 2-d)-6 f \hat{x}\} .
$$

Now suppose that both $\lambda=0$ and $\mu=0$. Then by (18)

$$
\hat{x}=\frac{g-3 f}{6 f}(1 / 2-d) .
$$

It is immediately clear that if $g<3 f$ condition (15) is violated. Substituting (19) into (17) and solving, one obtains

$$
d=\frac{g^{2}-f^{2}-2 g f}{2 g^{2}-6 f^{2}} \text { or } d=1 / 2 .
$$

After some manipulations it follows from (19) that if $d=\frac{g^{2}-f^{2}-2 g f}{2 g^{2}-6 f^{2}}$, then $p=(g-f) / 6$, and if $d=1 / 2$, then $p=(g-f) / 2$. These are two solution candidates in case $g \geq 3 f$, $\lambda=0$ and $\mu=0$.

Now suppose that $\lambda>0$, but $\mu=0$. Then $p=g d-f d$ by condition (15) and $\hat{x}=0$. By equations (17) and (18)

$$
\lambda=(1 / 2-d)^{2}-3 d \frac{g-f}{g}(1 / 2-d)=2 d(1 / 2-d)-3 d \frac{g-f}{g}(1 / 2-d) .
$$

Solving for $d$, one gets $d=1 / 2$ or $d=1 / 6$. If $d=1 / 2$, then $\lambda=0$, a contradiction, and if $d=1 / 6$ then $p=(g-f) / 6$. Note that for $d=1 / 6, \lambda>0$ if and only if $g<3 f$.

Now suppose that $\mu>0$, but $\lambda=0$. Then $d=1 / 2$ and by (18)

$$
\mu=-6 \frac{f}{g} p \hat{x}(p, 1 / 2) \leq 0 .
$$


This is a contradiction.

Finally, suppose that $\mu>0$ and $\lambda>0$. Then $d=1 / 2$ and $p=(g-f) / 2$. However, substituting these values into (17) one gets $\lambda=0$, again a contradiction.

Summarizing, if $g<3 f$, then the only solution candidate is $d=1 / 6$ and $p=(g-f) / 6$. If $g \leq 3 f$ there are two candidates, $d=1 / 2$ and $d=\frac{g^{2}-f^{2}-2 g f}{2 g^{2}-6 f^{2}}$. However, for $d=1 / 2$ and $p=(g-f) / 2$, profits are zero, while for $d=\frac{g^{2}-f^{2}-2 g f}{2 g^{2}-6 f^{2}}$ and $p=(g-f) / 6$ profits are strictly positive. Hence, $d=1 / 2$ and $p=(g-f) / 2$ can not be a maximum.

For each value of $g$ we now have a single remaining candidate that satisfies the first order conditions. Since a solution exists, this candidate must be the maximum. That is, if $g<3 f$, then $d^{M}=1 / 6$ and $p^{M}=(g-f) / 6$. If $g \leq 3 f$, then $d^{M}=\frac{g^{2}-f^{2}-2 g f}{2 g^{2}-6 f^{2}}$, and $p^{M}=(g-f) / 6$. Note that if $g=3 f$, then $d^{M}=1 / 6$. Q.E.D.

\section{References}

Borenstein, S. (1989), 'Hubs and high fares: dominance and market power in the u.s. airline industry', RAND Journal of Economics 20(3), 344-365.

Braid, R. M. (1989), 'The optimal locations of multiple bridges, connecting facilities, or product varieties', Journal of Regional Science 29(1), 63-70.

Crampton, G. R. (2000), 'Urban economic structure and the optimal rail system', Urban Studies 37(3), 623-632.

Hendricks, K., Piccione, M. \& Tan, G. (1995), 'The economics of hubs: The case of monopoly', Review Of Economic Studies 62(1), 83-99.

Hendricks, K., Piccione, M. \& Tan, G. (1997), 'Entry and exit in hub-spoke networks', Rand Journal Of Economics 28(2), 291-303.

Hyson, C. \& Hyson, W. (1950), 'The economic law of market areas', Quarterly Journal of Economics 64(2), 319-327. 
Niérat, P. (1997), 'Market area of rail-truck terminals: Pertinence of the spatial theory', Transportation Research A 31(2), 109-127.

Salop, S. C. (1979), 'Monopolistic competition with outside goods', Bell Journal of Economics 10(1), 141-156. 